the trace was compared with the sum of the computed eigenvalues, and found to differ by only a few units in the last place. file.

A copy of the B 5000 program and its output have been deposited in the UMT

Computer Science Division

Stanford University

Stanford, California 94305

1. B. Parlett, "Laguerre's method applied to the matrix eigenvalue problem," Math. Comp., v. 18, 1964, p. 469-485.

2. Burroughs Corporation, Equipment and Systems Marketing Division, "Extended Algol reference manual for the Burroughs B 5000," Detroit, 32, Michigan, November, 1962.

\title{
Differential Approximation Applied to the Solution of Convolution Equations
}

\section{By Richard Bellman, Robert Kalaba and Bella Kotkin}

1. Introduction. In the course of constructing some mathematical models of physiological processes connected with cancer chemotherapy [1], we have encountered functional equations containing convolution terms. Equations of this type are unpleasant computationally because of the storage, and thus time, requirements for solution. In some cases, these storage requirements could exceed present capabilities and thus seriously impede numerical solution.

We wish to present a new approach to this problem using the technique of differential approximation. To illustrate the method, we shall consider the equation

$$
u(t)=f(t)+\int_{0}^{t} e^{-(t-s)^{2}} u(s) d s
$$

2. Polynomial Approximation and Extensions. A classical problem, which owes its inception to a control process associated with the Watt steam engine (see [2]), is that of obtaining a polynomial which deviates the least from a given function, where the deviation is measured by an assigned norm.

If we recognize that a polynomial $p_{n}(t)=a_{0}+a_{1} t+\cdots+a_{n} t^{n}$ is a solution of the linear differential equation

$$
\frac{d^{(n+1)} u}{d t^{(n+1)}}=0
$$

then we see immediately that this problem is a particular case of the more general problem of finding an equation

$$
\frac{d^{(n+1)} u}{d t^{(n+1)}}+a_{1}(t) \frac{d^{n} u}{d t^{n}}+\cdots+a_{n}(t) u=0
$$

Received September 6, 1963. This investigation was supported in part by Public Health Service Research Grant Number RG-9608, from the Division of General Medical Sciences, National Institutes of Health. 
whose solution approximates to the given function in an optimal fashion. In the particular case where the $a_{i}(t)$ are constants, this is equivalent to asking for approximation by an exponential polynomial

$$
p_{n}(t)=\sum_{k=0}^{N} q_{k}(t) e^{\lambda_{k} t}
$$

This problem is in turn a special case of the general problem of approximating to a given function $f(t)$ by means of the solution of a nonlinear differential equation

$$
\frac{d^{(n+1)} u}{d t^{(n+1)}}=g\left(u, u^{\prime}, \cdots, u^{(n)}\right) .
$$

This is a meaningful approximation problem for arbitrary $n$, since an arbitrary analytic function will not in general satisfy a nonlinear differential equation of any finite order; e.g., $\Gamma(t)$.

This problem arises in the study of design and control and has an important role in the study of adaptive processes (see [3], [4]). We will discuss these matters elsewhere.

3. Linear Differential Approximation. We wish to consider the problem of approximating to a given function $f(t)$ by means of an exponential polynomial of the type appearing in (2.3), for reasons we shall describe below. Since a direct approach to this problem possesses well-known pitfalls (see Lanczos [5]), we shall pursue a different path.

First of all, we shall suppose that the given function $f(t)$ satisfies an ordinary differential equation

$$
f^{(m)}=h\left(f, f^{\prime}, \cdots, f^{(m-1)}, t\right),
$$

since this is quite often the case in applications.

Secondly, we shall determine an approximating linear differential equation with constant coefficients

$$
f^{(N)}+a_{1} f^{(N-1)}+\cdots+a_{N} f=0,
$$

by asking that the coefficients $a_{i}$ be chosen so as to minimize the functional

$$
\int_{0}^{T}\left(f^{(N)}+a_{1} f^{(N-1)}+\cdots+a_{N} f\right)^{2} d t
$$

where $f$ is determined by (3.1).

The approximation to $f$ will then be the solution of the linear differential equation

$$
u^{(N)}+a_{1} u^{(N-1)}+\cdots+a_{N} u=0,
$$

with initial conditions which will be determined in a fashion discussed below.

4. Computational Aspects. The minimization of the expression in (3.3) leads to the system of $N$ simultaneous linear equations

$$
\int_{0}^{T} f^{(j)} f^{(N)} d t+\sum_{i=1}^{N} a_{i}\left(\int_{0}^{T} f^{(N-i)} f^{(j)} d t\right)=0, \quad j=1,2, \cdots, N .
$$


For moderate values of $N$, i.e., $N \leqq 20$, the computational solution provides no difficulty once we have evaluated the integrals appearing as coefficients. We could, if we so desired, integrate by parts and reduce the evaluation of these integrals to the evaluation of the integrals $\int_{0}^{T}\left(f^{(i)}\right)^{2} d t$. For moderate size $N$, however, it is more convenient to proceed directly as follows.

Introduce new variables $u_{i j}, i, j=0,1, \cdots, N$, defined by the equations

$$
\frac{d u_{i j}}{d t}=f^{(N-i)} f^{(j)}, \quad u_{i j}(0)=0,
$$

and solve these equations simultaneously with the original equation for $f$, namely,

$$
f^{(m)}=h\left(f, f^{\prime}, \cdots, f^{(m-1)}, t\right) .
$$

5. Solution of Approximate Linear Equation. Having determined the coefficients $a_{i}$ by means of the foregoing procedures, we now wish to determine the function $u(t)$ as a solution of (3.4). A first approach is to use the initial values

$$
u^{(i)}(0)=f^{(i)}(0), \quad i=0,1,2, \cdots, N-1,
$$

and indeed this is what we do below with some success.

In general, however, we would proceed in the following fashion. Let $u_{1}, u_{2}, \cdots, u_{N}$ be the $N$ principal solutions of (3.4), the solutions determined by the condition that the matrix whose columns are $\left(u_{1}(0), u_{1}^{\prime}(0), \cdots, u_{1}^{(N-1)}(0)\right)$ etc., is the identity matrix.

Every solution can then be written in the form

$$
u(t)=\sum_{i=1}^{N} c_{i} u_{i}
$$

where the $c_{i}$ are scalars. Let us choose these $c_{i}$ so as to minimize the expression

$$
\int_{0}^{T}\left(f-\sum_{i=1}^{N} c_{i} u_{i}\right)^{2} d t
$$

The equations for the $c_{i}$ are

$$
\int_{0}^{T} f u_{i} d t-\sum_{j=1}^{N} c_{j} \int_{0}^{T} u_{i} u_{j} d t=0, \quad i=1,2, \cdots, N .
$$

To determine the various integrals, we introduce the variables $v_{i}$ and $w_{i j}$ by means of the relations

$$
\begin{aligned}
& \frac{d v_{i}}{d t}=f u_{i}, \quad v_{i}(0)=0 \\
& \frac{d w_{i j}}{d t}=u_{i} u_{j}, \quad w_{i j}(0)=0,
\end{aligned}
$$

adjoin the equations for the $u_{i}$ (equation (3.4) with appropriate boundary conditions) and the equation for $f$, and integrate.

6. An Example. Let us now discuss the equation of renewal type given in (1.1). Taking $0 \leqq t \leqq 1$, we obtain as a third-order differential approximation to the 
function $e^{-t^{2}}$ the solution of the equation

$$
u^{(3)}+2.740299 u^{(2)}+7.9511452 u^{(1)}+5.7636455 u=0 .
$$

Using the initial values obtained from $e^{-t^{2}}$, namely

$$
u(0)=1, \quad u^{\prime}(0)=0, \quad u^{\prime \prime}(0)=-2,
$$

we found such excellent agreement with the values of $e^{-t^{2}}$ over $0 \leqq t \leqq 1$ that there was no need to follow the procedure of Section 5 .

Consider the expression

$$
w(t)=\int_{0}^{t} k(t-s) u(s) d s .
$$

Differentiating repeatedly, and adding with the coefficients obtained above, we have

$$
\begin{aligned}
& w^{(3)}+2.740299 w^{(2)}+7.9511452 w^{(1)}+5.7636455 w \\
& =k(0) u^{\prime \prime}(t)+k^{\prime}(0) u^{\prime}(t)+k^{\prime \prime}(0) u(t) \\
& +2.740299\left[k(0) u^{\prime}(t)+k^{\prime}(0) u(t)\right]+7.9511452 k(0) u(t) \\
& +\int_{0}^{t} u(s)\left[k^{\prime \prime \prime}(t-s)+2.740299 k^{\prime \prime}(t-s)\right. \\
& \left.\quad+7.9511452 k^{\prime}(t-s)+5.7636455 k(t-s)\right] d s .
\end{aligned}
$$

Taking $k(t)=e^{-t^{2}}$ and assuming that the term under the integral sign is negligible, we obtain a third-order linear differential equation for $w=u-f$.

Let us take $f=1-\int_{0}^{t} e^{-s^{2}} d s$, so that the equation

$$
u(t)=1-\int_{0}^{t} e^{-s^{2}} d s+\int_{0}^{t} e^{-(t-s)^{2}} u(s) d s
$$

has the solution $u(t)=1$.

The function $f(t)$ as given above satisfies the linear differential equation

$$
f^{(3)}+2 t f^{(2)}+2 f^{(1)}=0,
$$

with $f(0)=1, f^{\prime}(0)=-1, f^{\prime \prime}(0)=0$.

Solving (6.6) together with the approximate linear equation for $u$ obtained from (6.4), we obtain the following values for $u(t)$ :

\begin{tabular}{c|c|c|c}
\hline$t$ & $u(t)$ & $u^{\prime}(t)$ & $u^{\prime \prime}(t)$ \\
\cline { 2 - 3 } 0.1 & 0.999999 & $\ldots$ & \\
0.2 & 0.999999 & $-0.14 \times 10^{-3}$ & $-0.148 \times 10^{-2}$ \\
0.3 & 0.999969 & $\ldots$ & $\ldots$ \\
0.4 & 0.999937 & $\ldots$ & $\cdots$ \\
0.5 & 0.999909 & $\ldots$ & $0.174 \times 10^{-3}$ \\
0.6 & 0.999898 & $0.229 \times 10^{-3}$ & $0.167 \times 10^{-3}$ \\
0.7 & 0.999909 & $0.330 \times 10^{-3}$ & $-0.135 \times 10^{-2}$ \\
0.8 & 0.999938 & $0.272 \times 10^{-3}$ & $-0.189 \times 10^{-2}$ \\
0.9 & 0.999970 & $0.919 \times 10^{-4}$ & -0.969 \\
1.0 & 0.99998 &
\end{tabular}


As we can see, the agreement with the desired value, $u(t)=1$, is excellent.

7. Discussion. Consider a system of renewal-type equations, given, say, in matrix form:

$$
X(t)=F(t)+\int_{0}^{t} K(t-s) X(s) d s .
$$

Equations of this type arise naturally in the study of multidimensional branching processes; see [6], [7].

If $X(t)$ is a $5 \times 5$ matrix, we are required to store 25 functions (i.e., the elements $\left.x_{i j}(t), i, j=1,2, \cdots, 5\right)$ if we proceed in the usual fashion. If high order accuracy were required-say, intervals of $10^{-3}$ over $0 \leqq t \leqq 5$-we would find that rapidaccess storage capacity would be exceeded.

On the other hand, if we use the foregoing technique, differential approximation of order 5 would lead to the task of solving about 250 simultaneous differential equations plus those required to determine $F(t)$. This is a simple matter for a modern computer. Furthermore, it is clear that we could use an approximation of order 10 without coming close to the storage capacity.

The RAND Corporation

Santa Monica, California

1. R. Bellman, J. Jacquez, R. Kalaba \& B. Kotkin, A Mathematical Model of Drug Distribution in the Body: Implications for Cancer Chemotherapy, The RAND Corporation Report No. RM-3463-NIH, February 1963.

2. R. Bellman \& R. Kalaba, Mathematical Trends in Control Theory, Dover Publications, New York. (To appear.)

3. R. Bellman, H. Kagiwada \& R. Kalaba, A Computational Procedure for Optimal System Design and Utilization, RAND Corporation Report No. RM-3174-PR, June 1962; also published in Proc. Nat. Acad. Sci. USA, V. 48, 1962, p. 1524-1528.

4. R. Bellman, "Mathematical model-making as an adaptive control process," Mathematical Optimization Techniques, University of California Press, Berkeley, 1963, p. 333-339.

5. C. Lanczos, Applied Analysis, Prentice-Hall, Englewood Cliffs, N. J., 1956.

6. T. E. Harris, Branching Processes, Ergebnisse der Math., Springer, Berlin, 1963.

7. R. Bellman \& K. L. Cooke, Differential-difference Equations, Academic Press, New York, 1963.

\section{On the Numerical Solution of Equations of the Abel Type}

\section{By Henry E. Fettis}

The integral equation known as Abel's has the general form

$$
f(x)=\int_{0}^{x} g(t)(x-t)^{-\alpha} d t
$$

where $\alpha$ is a real number, and

$$
0<\alpha<1 \text {. }
$$

Received August 20, 1963. 